\title{
Some inequalities for the multilinear singular integrals with Lipschitz functions on weighted Morrey spaces
}

Ferit Gürbüz ${ }^{1 *}$ (D)

"Correspondence:

feritgurbuz@hakkari.edu.tr

${ }^{1}$ Department of Mathematics

Education, Faculty of Education,

Hakkari University, Hakkari, Turkey

\begin{abstract}
The aim of this paper is to prove the boundedness of the oscillation and variation operators for the multilinear singular integrals with Lipschitz functions on weighted Morrey spaces.

MSC: 42B20; 42B25; 47G10

Keywords: Oscillation; Variation; Multilinear singular integral operators; Lipschitz space; Weighted Morrey space; Weights
\end{abstract}

\section{Introduction}

We first say that there exists a continuous function $K(x, y)$ defined on $\Omega=\{(x, y) \in \mathbb{R} \times \mathbb{R}$ : $x \neq y\}$ and $C>0$ if $K$ admits the following representation:

$$
|K(x, y)| \leq \frac{C}{|x-y|}, \quad \forall(x, y) \in \Omega,
$$

and for all $x, x_{0}, y \in \mathbb{R}$ with $|x-y|>2\left|x-x_{0}\right|$

$$
\begin{aligned}
& \left|K(x, y)-K\left(x_{0}, y\right)\right|+\left|K(y, x)-K\left(y, x_{0}\right)\right| \\
& \quad \leq \frac{C}{|x-y|}\left(\frac{\left|x-x_{0}\right|}{|x-y|}\right)^{\beta},
\end{aligned}
$$

where $1>\beta>0$. Then $K$ is said to be a Calderón-Zygmund standard kernel.

Suppose that $K$ satisfies (1) and (2). Then Zhang and Wu [12] considered the family of operators $T:=\left\{T_{\epsilon}\right\}_{\epsilon>0}$ and a related the family of commutator operators $T_{b}:=\left\{T_{\epsilon, b}\right\}_{\epsilon>0}$ generated by $T_{\epsilon}$ and $b$ which are given by

$$
T_{\epsilon} f(x)=\int_{|x-y|>\epsilon} K(x, y) f(y) d y
$$

(c) The Author(s) 2020. This article is licensed under a Creative Commons Attribution 4.0 International License, which permits use, sharing, adaptation, distribution and reproduction in any medium or format, as long as you give appropriate credit to the original author(s) and the source, provide a link to the Creative Commons licence, and indicate if changes were made. The images or other third party material in this article are included in the article's Creative Commons licence, unless indicated otherwise in a credit line to the material. If material is not included in the article's Creative Commons licence and your intended use is not permitted by statutory regulation or exceeds the permitted use, you will need to obtain permission directly from the copyright holder. To view a copy of this licence, visit http://creativecommons.org/licenses/by/4.0/. 
and

$$
T_{\epsilon, b} f(x)=\int_{|x-y|>\epsilon}(b(x)-b(y)) K(x, y) f(y) d y .
$$

In this sense, following [12], the definition of the oscillation operator of $T$ is given by

$$
\mathcal{O}(T f)(x):=\left(\sum_{i=1}^{\infty} \sup _{t_{i+1} \leq \epsilon_{i+1}<\epsilon_{i} \leq t_{i}}\left|T_{\epsilon_{i+1}} f(x)-T_{\epsilon_{i}} f(x)\right|^{2}\right)^{\frac{1}{2}}
$$

where $\left\{t_{i}\right\}$ is a decreasing fixed sequence of positive numbers converging to 0 and a related $\rho$-variation operator is defined by

$$
\mathcal{V}_{\rho}(T f)(x):=\sup _{\epsilon_{i} \searrow 0}\left(\sum_{i=1}^{\infty}\left|T_{\epsilon_{i+1}} f(x)-T_{\epsilon_{i}} f(x)\right|^{\rho}\right)^{\frac{1}{\rho}}, \quad \rho>2,
$$

where the supremum is taken over all sequences of real number $\left\{\epsilon_{i}\right\}$ decreasing to 0 . We also take into account the operator

$$
\mathcal{O}^{\prime}(T f)(x):=\left(\sum_{i=1}^{\infty} \sup _{t_{i+1}<\eta_{i}<t_{i}}\left|T_{t_{i+1}} f(x)-T_{\eta_{i}} f(x)\right|^{2}\right)^{\frac{1}{2}}
$$

On the other hand, it is obvious that

$$
\mathcal{O}^{\prime}(T f) \approx \mathcal{O}(T f)
$$

That is,

$$
\mathcal{O}^{\prime}(T f) \leq \mathcal{O}(T f) \leq 2 \mathcal{O}^{\prime}(T f)
$$

Recently, Campbell et al. in [1] proved the oscillation and variation inequalities for the Hilbert transform in $L^{p}(1<p<\infty)$ and then following [1], we denote by $E$ the mixed norm Banach space of the two-variable function $h$ defined on $\mathbb{R} \times \mathbb{N}$ such that

$$
\|h\|_{E} \equiv\left(\sum_{i}\left(\sup _{s}|h(s, i)|\right)^{2}\right)^{1 / 2}<\infty
$$

Given $T:=\left\{T_{\epsilon}\right\}_{\epsilon>0}$ is a family operators such that $\lim _{\epsilon \rightarrow 0} T_{\epsilon} f(x)=T f(x)$ exists almost everywhere for certain class of functions $f$, where $T_{\epsilon}$ defined as (3). For a fixed decreasing sequence $\left\{t_{i}\right\}$ with $t_{i} \searrow 0$, let $J_{i}=\left(t_{i+1}, t_{i}\right]$ and define the $E$-valued operator $U(T): f \rightarrow U(T) f$ given by

$$
U(T) f(x)=\left\{T_{t_{i+1}} f(x)-T_{s} f(x)\right\}_{s \in J_{i}, i \in \mathbb{N}}=\left\{\int_{\left\{t_{i+1}<|x-y|<s\right\}} K(x, y) f(y) d y\right\}_{s \in J_{i}, i \in \mathbb{N}}
$$


Then

$$
\begin{aligned}
\mathcal{O}^{\prime}(T f)(x) & =\|U(T) f(x)\|_{E}=\left\|\left\{T_{t_{i+1}} f(x)-T_{s} f(x)\right\}_{s \in J_{i}, i \in \mathbb{N}}\right\|_{E} \\
& =\left\|\left\{\int_{\left\{t_{i+1}<|x-y|<s\right\}} K(x, y) f(y) d y\right\}_{s \in J_{i}, i \in \mathbb{N}}\right\|_{E} .
\end{aligned}
$$

Let $\Phi=\left\{\beta: \beta=\left\{\epsilon_{i}\right\}, \epsilon_{i} \in \mathbb{R}, \epsilon_{i} \searrow 0\right\}$. We denote by $F_{\rho}$ the mixed norm space of twovariable functions $g(i, \beta)$ such that

$$
\|g\|_{F_{\rho}} \equiv \sup _{\beta}\left(\sum_{i}|g(i, \beta)|^{\rho}\right)^{1 / \rho}
$$

We also take into account the $F_{\rho}$-valued operator $V(T): f \rightarrow V(T) f$ such that

$$
V(T) f(x)=\left\{T_{\epsilon_{i+1}} f(x)-T_{\epsilon_{i}} f(x)\right\}_{\beta=\left\{\epsilon_{i}\right\} \in \Phi} .
$$

Thus,

$$
V_{\rho}(T) f(x)=\|V(T) f(x)\|_{F_{\rho}}
$$

Given $m$ is a positive integer, and $b$ is a function on $\mathbb{R}$. Let $R_{m+1}(b ; x, y)$ be the $m+1$ th order Taylor series remainder of $b$ at $x$ about $y$, that is,

$$
R_{m+1}(b ; x, y)=b(x)-\sum_{\gamma \leq m} \frac{1}{\gamma !} b^{(\gamma)}(y)(x-y)^{\gamma} .
$$

In this paper, we consider the family of operators $T^{b}:=\left\{T_{\epsilon}^{b}\right\}_{\epsilon>0}$ given by [6], where $T_{\epsilon}^{b}$ are the multilinear singular integral operators of $T_{\epsilon}$ as follows:

$$
T_{\epsilon}^{b} f(x)=\int_{|x-y|>\epsilon} \frac{R_{m+1}(b ; x, y)}{|x-y|^{m}} K(x, y) f(y) d y .
$$

Thus, if $m=0$, then $T_{\epsilon}^{b}$ is just the commutator of $T_{\epsilon}$ and $b$, which is given by (4). But, if $m>0$, then $T_{\epsilon}^{b}$ are non-trivial generation of the commutators.

The theory of multilinear analysis was received extensive studies in the last 3 decades (see $[2,5]$ for example). Hu and Wang [6] proved that the weighted $\left(L^{p}, L^{q}\right)$-boundedness of the oscillation and variation operators for $T^{b}$ when the $m$ th derivative of $b$ belongs to the homogeneous Lipschitz space $\dot{\Lambda}_{\beta}$. In this sense, we recall the definition of homogeneous Lipschitz space $\dot{\Lambda}_{\beta}$ as follows.

Definition 1 (Homogeneous Lipschitz space) Let $0<\beta \leq 1$. The homogeneous Lipschitz space $\dot{\Lambda}_{\beta}$ is defined by

$$
\dot{\Lambda}_{\beta}(\mathbb{R})=\left\{b:\|b\|_{\dot{\Lambda}_{\beta}}=\sup _{x, h \in \mathbb{R}, h \neq 0} \frac{|b(x+h)-b(x)|}{|h|^{\beta}}<\infty\right\} .
$$

Obviously, if $\beta>1$, then $\dot{\Lambda}_{\beta}(\mathbb{R})$ only includes constant. So we restrict $0<\beta \leq 1$. 
Now, we recall the definitions of basic spaces such as Morrey, weighted Lebesgue, weighted Morrey spaces and consider the relationship between these spaces.

Besides the Lebesgue space $L^{q}(\mathbb{R})$, the Morrey space $M_{p}^{q}(\mathbb{R})$ is another important function space with definition as follows.

Definition 2 (Morrey space) For $1 \leq p \leq q<\infty$, the Morrey space $M_{p}^{q}(\mathbb{R})$ is the collection of all measurable functions $f$ whose Morrey space norm is

$$
\|f\|_{M_{p}^{q}(\mathbb{R})}=\sup _{\substack{I \subset \mathbb{R} \\ I: \text { Interval }}} \frac{1}{|I|^{\frac{1}{p}-\frac{1}{q}}}\left\|f \chi_{I}\right\|_{L_{p}(\mathbb{R})}<\infty .
$$

Remark 1 If $p=q$, then

$$
\|f\|_{M_{q}^{q}(\mathbb{R})}=\|f\|_{L^{q(\mathbb{R})}}
$$

If $q<p$, then $M_{p}^{q}(\mathbb{R})$ is strictly larger than $L^{q}(\mathbb{R})$. For example, $f(x):=|x|^{-\frac{1}{q}} \in M_{p}^{q}(\mathbb{R})$ but $f(x):=|x|^{-\frac{1}{q}} \notin L^{q}(\mathbb{R})$.

On the other hand, for a given weight function $w$ and any interval $I$, we also denote the Lebesgue measure of $I$ by $|I|$ and set weighted measure

$$
w(I)=\int_{I} w(x) d x
$$

For $0<p<\infty$, the weighted Lebesgue space $L_{p}(w) \equiv L_{p}(\mathbb{R}, w)$ is defined by the norm

$$
\|f\|_{L_{p}(w)}=\left(\int_{\mathbb{R}}|f(x)|^{p} w(x) d x\right)^{\frac{1}{p}}<\infty .
$$

A weight $w$ is said to belong to the Muckenhoupt class $A_{p}$ for $1<p<\infty$ such that

$$
\begin{aligned}
{[w]_{A_{p}}:=\sup _{I}[w]_{A_{p}(I)} } \\
\quad=\sup _{I}\left(\frac{1}{|I|} \int_{I} w(x) d x\right)\left(\frac{1}{|I|} \int_{I} w(x)^{1-p^{\prime}} d x\right)^{p-1}<\infty,
\end{aligned}
$$

where $p^{\prime}=\frac{p}{p-1}$. The condition (6) is called the $A_{p}$-condition, and the weights which satisfy it are called $A_{p}$-weights. The expression $[w]_{A_{p}}$ is also called characteristic constant of $w$.

Here and after, $A_{p}$ denotes the Muckenhoupt classes (see $\left.[5,7]\right)$. The $A_{p}$ class of weights characterizes the $L_{p}(w)$ boundedness of the maximal function as Muckenhoupt [9] established in the 1970s. Subsequent work of Muckenhoupt [9] himself Muckenhoupt and Wheeden [10,11], Coifman and Fefferman [3] was devoted to exploring the connection of the $A_{p}$ class with weighted estimates for singular integrals. However, it was not until the 2000s that the quantitative dependence on the so called $A_{p}$ constant, namely $[w]_{A_{p}}$, became a trending topic.

When $p=1, w \in A_{1}$ if there exists $C>1$ such that, for almost every $x$,

$$
M w(x) d x \leq C w(x)
$$


and the infimum of $C$ satisfying the inequality (7) is denoted by $[w]_{A_{1}}$, where $M$ is the classical Hardy-Littlewood maximal operator.

When $p=\infty$, we define $A_{\infty}(\mathbb{R})=\bigcup_{1 \leq p<\infty} A_{p}(\mathbb{R})$. That is, the $A_{\infty}$ constant is given by

$$
\begin{aligned}
{[w]_{A_{\infty}}: } & =\sup _{I}[w]_{A_{\infty}(I)} \\
& =\sup _{I} \int_{I} M\left(\chi_{I} w\right)(x) d x,
\end{aligned}
$$

where we utilize the notation $M\left(\chi_{I} w\right)$ to denote the Hardy-Littlewood maximal function of a function $\chi_{I} w$ by

$$
M\left(\chi_{I} w\right)(x):=\sup _{I} \frac{1}{|I|} \int_{I}\left|\chi_{I} w(x)\right| d x .
$$

A weight function $w$ belongs to $A_{p, q}$ (Muckenhoupt-Wheeden class) for $1<p<q<\infty$ if

$$
\begin{aligned}
{[w]_{A_{p, q}}:=\sup _{I}[w]_{A_{p, q}(I)} } \\
\quad=\sup _{I}\left(\frac{1}{|I|} \int_{I} w(x)^{q} d x\right)^{\frac{1}{q}}\left(\frac{1}{|I|} \int_{I} w(x)^{-p^{\prime}} d x\right)^{\frac{1}{p^{\prime}}}<\infty .
\end{aligned}
$$

From the definition of $A_{p, q}$, we know that $w(x) \in A_{p, q}(\mathbb{R})$ implies $w(x)^{q} \in A_{q}(\mathbb{R})$ and $w(x)^{p} \in$ $A_{p}(\mathbb{R})$.

Now, we begin with some lemmas. These lemmas are very necessary for the proof of the main result.

Lemma 1 ([4]) If $w \in A_{p}, p \geq 1$, then there exists a constant $C>0$ such that

$$
w(2 I) \leq C w(I) .
$$

for any interval $I$.

More precisely, for all $\lambda>1$ we have

$$
w(\lambda I) \leq C \lambda^{p} w(I)
$$

where $C$ is a constant independent of $I$ or $\lambda$ and $w(I)=\int_{I} w(x) d x$.

Lemma 2 ([2]) Let $b$ be a function on $\mathbb{R}$ and $b^{(m)} \in L_{u}(\mathbb{R})$ with $m \in \mathbb{N}$ for any $u>1$. Then

$$
\left|R_{m}(b ; x, y)\right| \leq C|x-y|^{m}\left(\frac{1}{|I(x, y)|} \int_{I(x, y)}\left|b^{(m)}(z)\right|^{u} d z\right)^{\frac{1}{u}}, \quad C>0,
$$

where $I(x, y)$ is the interval $(x-5|x-y|, x+5|x-y|)$.

Lemma 3 ([6]) Let $K(x, y)$ satisfies (1) and (2), $\rho>2$, and $T:=\left\{T_{\epsilon}\right\}_{\epsilon>0}$ and $T^{b}:=\left\{T_{\epsilon}^{b}\right\}_{\epsilon>0}$ be given by (3) and (5), respectively. If $\mathcal{O}(T)$ and $\mathcal{V}_{\rho}(T)$ are bounded on $L_{p_{0}}(\mathbb{R}, d x)$ for some 
$1<p_{0}<\infty$, and $b^{(m)} \in \dot{\Lambda}_{\beta}$ with $m \in \mathbb{N}$ for $0<\beta<1$, then

$$
\left\|\mathcal{O}^{\prime}\left(T^{b}\right)\right\|_{L_{q}\left(w^{q}\right)} \leq\left\|\mathcal{O}\left(T^{b}\right)\right\|_{L_{q}\left(w^{q}\right)} \leq C\|b\|_{\dot{\Lambda}_{\beta}}\|f\|_{L_{p}\left(w^{p}\right)}, \quad C>0,
$$

and

$$
\left\|\mathcal{V}_{\rho}\left(T^{b}\right)\right\|_{L_{q}\left(w^{q}\right)} \leq C\|b\|_{\dot{\Lambda}_{\beta}}\|f\|_{L_{p}\left(w^{p}\right)}, \quad C>0
$$

for any $1<p<\frac{1}{\beta}$ with $\frac{1}{q}=\frac{1}{p}-\beta$ and $w \in A_{p, q}$.

Next, in 2009, the weighted Morrey space $L_{p, \kappa}(w)$ was defined by Komori and Shirai [7] as follows.

Definition 3 (Weighted Morrey space) Let $1 \leq p<\infty, 0<\kappa<1$ and $w$ be a weight function. Then the weighted Morrey space $L_{p, \kappa}(w) \equiv L_{p, \kappa}(\mathbb{R}, w)$ is defined by

$$
L_{p, \kappa}(w) \equiv L_{p, \kappa}(\mathbb{R}, w)=\left\{f \in L_{p, w}^{\mathrm{loc}}(\mathbb{R}):\|f\|_{L_{p, \kappa}(w)}=\sup _{I} w(I)^{-\frac{\kappa}{p}}\|f\|_{L_{p, w}(I)}<\infty\right\}
$$

Remark 2 If $\kappa=0$, then

$$
\|f\|_{L_{p, 0}(w)}=\|f\|_{L_{p}(w)}
$$

When $w \equiv 1$ and $\kappa=1-\frac{p}{q}$ with $1<p \leq q<\infty$, then

$$
\|f\|_{L_{p, 1-\frac{p}{q}}(1)}=\|f\|_{M_{p}^{q}(\mathbb{R})}
$$

Finally, we recall the definition of the weighted Morrey space with two weights as follows.

Definition 4 (Weighted Morrey space with two weights) Let $1 \leq p<\infty$ and $0<\kappa<1$. Then for two weights $u$ and $v$, the weighted Morrey space $L_{p, \kappa}(u, v) \equiv L_{p, \kappa}(\mathbb{R}, u, v)$ is defined by

$$
L_{p, \kappa}(u, v) \equiv L_{p, \kappa}(\mathbb{R}, u, v)=\left\{f \in L_{p, u}^{\mathrm{loc}}(\mathbb{R}):\|f\|_{L_{p, \kappa}(w)}=\sup _{I} v(I)^{-\frac{\kappa}{p}}\|f\|_{L_{p, u}(I)}<\infty\right\}
$$

It is obvious that

$$
L_{p, \kappa}(w, w) \equiv L_{p, \kappa}(w)
$$

In 2016, Zhang and Wu [12] gave the boundedness of the oscillation and variation operators for Calderón-Zygmund singular integrals and the corresponding commutators on the weighted Morrey spaces. In 2017, Hu and Wang [6] established the weighted $\left(L^{p}, L^{q}\right)$ inequalities of the variation and oscillation operators for the multilinear CalderónZygmund singular integral with a Lipschitz function in $\mathbb{R}$. Inspired of these results $[6,12]$, we investigate the boundedness of the oscillation and variation operators for the family of 
the multilinear singular integral defined by (5) on weighted Morrey spaces when the $m$ th derivative of $b$ belongs to the homogeneous Lipschitz space $\dot{\Lambda}_{\beta}$ in this work.

Throughout this paper, $C$ always means a positive constant independent of the main parameters involved, and may change from one occurrence to another. We also use the notation $F \lesssim G$ to mean $F \leq C G$ for an appropriate constant $C>0$, and $F \approx G$ to mean $F \lesssim G$ and $G \lesssim F$.

\section{Main result}

We now formulate our main result as follows.

Theorem 1 Let $K(x, y)$ satisfies (1) and (2), $\rho>2$, and $T:=\left\{T_{\epsilon}\right\}_{\epsilon>0}$ and $T^{b}:=\left\{T_{\epsilon}^{b}\right\}_{\epsilon>0}$ be given by (3) and (5), respectively. If $\mathcal{O}(T)$ and $\mathcal{V}_{\rho}(T)$ are bounded on $L_{p_{0}}(\mathbb{R}, d x)$ for some $1<p_{0}<\infty$, and $b^{(m)} \in \dot{\Lambda}_{\beta}$ with $m \in \mathbb{N}$ for $0<\beta<1$, then $\mathcal{O}\left(T^{b}\right)$ and $\mathcal{V}_{\rho}\left(T^{b}\right)$ are bounded from $L_{p, \kappa}\left(w^{p}, w^{q}\right)$ to $L_{p, \frac{\kappa q}{p}}\left(w^{q}\right)$ for any $1<p<\frac{1}{\beta}, \frac{1}{q}=\frac{1}{p}-\beta, 0<\kappa<\frac{p}{q}$ and $w \in A_{p, q}$.

Corollary 1 ([12]) Let $K(x, y)$ satisfies (1) and (2), $\rho>2$, and $T:=\left\{T_{\epsilon}\right\}_{\epsilon>0}$ and $T_{b}:=$ $\left\{T_{\epsilon, b}\right\}_{\epsilon>0}$ be given by (3) and (4), respectively. If $\mathcal{O}(T)$ and $\mathcal{V}_{\rho}(T)$ are bounded on $L_{p_{0}}(\mathbb{R}, d x)$ for some $1<p_{0}<\infty$, and $b \in \dot{\Lambda}_{\beta}$ for $0<\beta<1$, then $\mathcal{O}\left(T_{b}\right)$ and $\mathcal{V}_{\rho}\left(T_{b}\right)$ are bounded from $L_{p, \kappa}\left(w^{p}, w^{q}\right)$ to $L_{p, \frac{\kappa q}{p}}\left(w^{q}\right)$ for any $1<p<\frac{1}{\beta}, \frac{1}{q}=\frac{1}{p}-\beta, 0<\kappa<\frac{p}{q}$ and $w \in A_{p, q}$.

\subsection{The proof of Theorem 1}

Proof We consider the proof related to $\mathcal{O}\left(T^{b}\right)$ firstly. Fix an interval $I=\left(x_{0}-l, x_{0}+l\right)$, and we write as $f=f_{1}+f_{2}$, where $f_{1}=f \chi_{2 I}, \chi_{2 I}$ denotes the characteristic function of $2 I$. Thus, it is sufficient to show that the conclusion

$$
\begin{aligned}
\left\|\mathcal{O}^{\prime}\left(T^{b} f\right)(x)\right\|_{L_{p, \frac{k q}{p}\left(w^{q}\right)}} & \leq\left\|\mathcal{O}^{\prime}\left(T^{b} f_{1}\right)(x)\right\|_{L_{p, \frac{\kappa q}{p}\left(w^{q}\right)}}+\left\|\mathcal{O}^{\prime}\left(T^{b} f_{1}\right)(x)\right\|_{L_{p, \frac{\kappa q}{p}\left(w^{q}\right)}} \\
& \lesssim\|b\|_{\dot{\Lambda}_{\beta}}\|f\|_{L_{p, \kappa}\left(w^{p}, w^{q}\right)}
\end{aligned}
$$

holds for every interval $I \subset \mathbb{R}$. Then

$$
\begin{aligned}
& \left(\int_{I}\left|\mathcal{O}^{\prime}\left(T^{b} f\right)(x)\right|^{q} w^{q}(x) d x\right)^{\frac{1}{q}} \\
& \quad \leq\left(\int_{I}\left|\mathcal{O}^{\prime}\left(T^{b} f_{1}\right)(x)\right|^{q} w^{q}(x) d x\right)^{\frac{1}{q}}+\left(\int_{I}\left|\mathcal{O}^{\prime}\left(T^{b} f_{2}\right)(x)\right|^{q} w^{q}(x) d x\right)^{\frac{1}{q}} \\
& \quad=: F_{1}+F_{2} .
\end{aligned}
$$

First, we use (9) to estimate $F_{1}$, and we obtain

$$
\begin{aligned}
F_{1} & =\left(\int_{I}\left|\mathcal{O}^{\prime}\left(T^{b} f_{1}\right)(x)\right|^{q} w^{q}(x) d x\right)^{\frac{1}{q}} \lesssim\|b\|_{\dot{A}_{\beta}}\left\|f_{1}\right\|_{L_{p}\left(w^{p}\right)} \\
& =\|b\|_{\dot{A}_{\beta}}\left(\frac{1}{w^{q}(2 I)^{\kappa}} \int_{2 I}|f(x)|^{p} w^{p}(x) d x\right)^{\frac{1}{p}} w^{q}(2 I)^{\frac{\kappa}{p}} \\
& \lesssim\|b\|_{\dot{A}_{\beta}}\|f\|_{L_{p, \kappa}\left(w^{p}, w^{q}\right)^{q}}^{p} w^{q}(I)^{\frac{\kappa}{p}} .
\end{aligned}
$$


Thus,

$$
\left\|\mathcal{O}^{\prime}\left(T^{b} f_{1}\right)(x)\right\|_{L_{p, \frac{\kappa q}{p}\left(w^{q}\right)}} \lesssim\|b\|_{\dot{\Lambda}_{\beta}}\|f\|_{L_{p, \kappa}\left(w^{p}, w^{q}\right)}
$$

Second, for $x \in I, k=1,2, \ldots, m \in \mathbb{N}$, let $A_{k}=\left\{y: 2^{k} l \leq|y-x|<2^{k+1} l\right\}, B_{k}=\{y:|y-x|<$ $\left.2^{k+1} l\right\}$, and

$$
b_{k}(z)=b(z)-\frac{1}{m !}\left(b^{(m)}\right)_{B_{k}} z^{m}
$$

By [2], for any $y \in A_{k}$, it is obvious that

$$
R_{m+1}(b ; x, y)=R_{m+1}\left(b_{k} ; x, y\right) .
$$

Moreover, since $b \in \dot{\Lambda}_{\beta}$, for $y \in A_{k}$, we get

$$
\begin{aligned}
\left|b^{(m)}(y)-\left(b^{(m)}\right)_{B_{k}}\right| & \leq \frac{1}{\left|B_{k}\right|} \int_{B_{k}}\left|b^{(m)}(y)-b^{(m)}(z)\right| d z \\
& \lesssim\left\|b^{(m)}\right\|_{\dot{\Lambda}_{\beta}}\left(2^{k} l\right)^{\beta} .
\end{aligned}
$$

Hence, by Lemma 2 and (11)

$$
\begin{aligned}
R_{m}\left(b_{k} ; x, y\right) & \lesssim|x-y|^{m}\left(\frac{1}{|I(x, y)|} \int_{I(x, y)}\left|b^{(m)}(z)\right|^{u} d z\right)^{\frac{1}{u}} \\
& \lesssim|x-y|^{m}\left\|b^{(m)}\right\|_{\dot{\Lambda}_{\beta}}\left(2^{k} l\right)^{\beta} .
\end{aligned}
$$

Also, following [12], we have

$$
\left\|\left\{\chi_{\left\{t_{i}+1<|x-y|<u\right\}}\right\}_{u \in J_{i}, i \in \mathbb{N}}\right\|_{A} \leq 1 .
$$

Thus, the estimate of $F_{2}$ can be obtained as follows:

$$
\begin{aligned}
\left|\mathcal{O}^{\prime}\left(T^{b} f_{2}\right)(x)\right| & =\left\|U\left(T^{b} f_{2}\right)(x)\right\| \\
& =\left\|\left\{\int_{\left\{t_{i}+1<|x-y|<u\right\}} \frac{R_{m+1}(b ; x, y)}{|x-y|^{m}} K(x, y) f_{2}(y) d y\right\}\right\|_{A} \\
& \leq \int_{\mathbb{R}}\left\|\left\{\chi_{\left\{t_{i}+1<|x-y|<u\right\}}\right\}_{u \in J_{i}, i \in \mathbb{N}}\right\|_{A}\left|\frac{R_{m+1}(b ; x, y)}{|x-y|^{m}} K(x, y) f_{2}(y)\right| d y \\
& \leq \int_{\mathbb{R}}\left|\frac{R_{m+1}(b ; x, y)}{|x-y|^{m}} K(x, y) f_{2}(y)\right| d y \\
& \lesssim \int_{|x-y|>2 l} \frac{\mid R_{m+1}(b ; x, y)}{|x-y|^{m}} K(x, y) f(y) \mid d y \\
& \lesssim \sum_{k=1}^{\infty} \frac{1}{2^{k} l} \int_{A_{k}}\left(\left\|b^{(m)}\right\|_{\dot{\Lambda}_{\beta}}\left(2^{k} l\right)^{\beta}+\left|b^{(m)}(y)-\left(b^{(m)}\right)_{B_{k}}\right|\right)|f(y)| d y \\
& \lesssim\left\|b^{(m)}\right\|_{\dot{\Lambda}_{\beta}} \sum_{k=1}^{\infty} \frac{1}{\left(2^{k} l\right)^{1-\beta}} \int_{A_{k}}|f(y)| d y
\end{aligned}
$$




$$
\begin{aligned}
& +\sum_{k=1}^{\infty} \frac{1}{2^{k} l} \int_{A_{k}}\left|b^{(m)}(y)-\left(b^{(m)}\right)_{B_{k}}\right||f(y)| d y \\
= & G_{1}+G_{2} .
\end{aligned}
$$

For $G_{1}$, since

$$
\left(\int_{A_{k}} w(y)^{-p^{\prime}} d y\right)^{\frac{1}{p^{\prime}}} \lesssim w^{q}\left(B_{k}\right)^{-\frac{1}{q}}\left|B_{k}\right|^{\frac{1}{p^{\prime}}+\frac{1}{q}}
$$

with $1<p<\frac{1}{\beta}, \frac{1}{q}=\frac{1}{p}-\beta$ and using Hölder's inequality, we have

$$
\begin{aligned}
& \sum_{k=1}^{\infty} \frac{1}{\left(2^{k} l\right)^{1-\beta}} \int_{A_{k}}|f(y)| d y \\
& \quad \lesssim \sum_{k=1}^{\infty} \frac{1}{\left(2^{k} l\right)^{1-\beta}}\left(\int_{A_{k}}|f(y)|^{p} w^{p}(y) d y\right)^{\frac{1}{p}}\left(\int_{A_{k}} w(y)^{-p^{\prime}} d y\right)^{\frac{1}{p^{\prime}}} \\
& \quad \lesssim\|f\|_{L_{p, \kappa}\left(w^{p}, w^{q}\right)} \sum_{k=1}^{\infty} \frac{\left(2^{k} l\right)^{\frac{1}{p^{\prime}}+\frac{1}{q}}}{\left(2^{k} l\right)^{1-\beta}} w^{q}\left(B_{k}\right)^{\frac{\kappa}{p}-\frac{1}{q}} \\
& \quad \lesssim\|f\|_{L_{p, \kappa}\left(w^{p}, w^{q}\right)} \sum_{k=1}^{\infty} w^{q}\left(B_{k}\right)^{\frac{\kappa}{p}-\frac{1}{q}}
\end{aligned}
$$

Since $w \in A_{p, q}$, we have $w^{q} \in A_{\infty}$. Thus, Lemma 1 implies $w^{q}\left(B_{k}\right) \leq(C)^{k} w^{q}(I), C>1$, i.e.,

$$
\sum_{k=1}^{\infty} w^{q}\left(B_{k}\right)^{\frac{\kappa}{p}-\frac{1}{q}} \lesssim w^{q}(I)^{\frac{\kappa}{p}-\frac{1}{q}} \sum_{k=1}^{\infty} C^{\frac{\kappa}{p}-\frac{1}{q}} \lesssim w^{q}(I)^{\frac{\kappa}{p}-\frac{1}{q}}
$$

with $\frac{\kappa}{p}-\frac{1}{q}<0$. This implies

$$
G_{1} \lesssim\left\|b^{(m)}\right\|_{\dot{\Lambda}_{\beta}}\|f\|_{L_{p, \kappa}\left(w^{p}, w^{q}\right)} w^{q}(I)^{\frac{\kappa}{p}-\frac{1}{q}} .
$$

Let $y \in A_{k}$. For $G_{2}$, by (11), (12) and (13) we get

$$
\begin{aligned}
G_{2} & \lesssim\left\|b^{(m)}\right\|_{\dot{\Lambda}_{\beta}} \sum_{k=1}^{\infty} \frac{1}{\left(2^{k+1} l\right)^{1-\beta}} \int_{A_{k}}|f(y)| d y \\
& \lesssim\left\|b^{(m)}\right\|_{\dot{A}_{\beta}}\|f\|_{L_{p, \kappa}\left(w^{p}, w^{q}\right)} w^{q}(I)^{\frac{\kappa}{p}-\frac{1}{q}} .
\end{aligned}
$$

Thus, by (14) and (15), we obtain

$$
\begin{aligned}
F_{2} & =\left(\int_{I}\left|\mathcal{O}^{\prime}\left(T^{b} f_{2}\right)(x)\right|^{q} w^{q}(x) d x\right)^{\frac{1}{q}} \\
& \lesssim\left\|b^{(m)}\right\|_{\dot{\Lambda}_{\beta}}\|f\|_{L_{p, \kappa}\left(w^{p}, w^{q}\right)} w^{q}(I)^{\frac{\kappa}{p}-\frac{1}{q}} w^{q}(I)^{\frac{1}{q}} \\
& =\left\|b^{(m)}\right\|_{\dot{\Lambda}_{\beta}}\|f\|_{L_{p, \kappa}\left(w^{p}, w^{q}\right)} w^{q}(I)^{\frac{\kappa}{p}} .
\end{aligned}
$$


Thus,

$$
\left\|\mathcal{O}^{\prime}\left(T^{b} f_{2}\right)(x)\right\|_{L_{p, \frac{\kappa q}{p}\left(w^{q}\right)}} \lesssim\|b\|_{\dot{\Lambda}_{\beta}}\|f\|_{L_{p, \kappa}\left(w^{p}, w^{q}\right)} .
$$

As a result, by (10) and (16), we get

$$
\left\|\mathcal{O}^{\prime}\left(T^{b} f\right)(x)\right\|_{L_{p, \frac{\kappa q}{p}\left(w^{q}\right)}} \lesssim\|b\|_{\dot{\Lambda}_{\beta}}\|f\|_{L_{p, \kappa}\left(w^{p}, w^{q}\right)} .
$$

Similarly, $\mathcal{V}_{\rho}\left(T^{b}\right)$ has the same estimate as above (here we omit the details), thus the inequality

$$
\left\|\mathcal{V}_{\rho}\left(T^{b} f\right)(x)\right\|_{L_{p, \frac{\kappa q}{p}\left(w^{q}\right)}} \lesssim\|b\|_{\dot{\Lambda}_{\beta}}\|f\|_{L_{p, \kappa}\left(w^{p}, w^{q}\right)}
$$

is valid.

Therefore, Theorem 1 is completely proved.

\section{Acknowledgements}

The author thanks the referees for their numerous helpful suggestions.

\section{Funding}

No funding is used to support this paper.

Availability of data and materials

Not applicable.

\section{Competing interests}

The author declares that he has no competing interests.

\section{Authors' contributions}

The author was the only one to contribute to the writing of this paper. He read and approved the manuscript.

\section{Publisher's Note}

Springer Nature remains neutral with regard to jurisdictional claims in published maps and institutional affiliations.

Received: 29 January 2020 Accepted: 29 April 2020 Published online: 11 May 2020

References

1. Campbell, J.T., Jones, R.L., Reinhold, K., Wierdl, M.: Oscillation and variation for the Hilbert transform. Duke Math. J. $105,59-83(2000)$

2. Cohen, J., Gosselin, J.: A BMO estimate for multilinear singular integrals. III. J. Math. 30, 445-464 (1986)

3. Coifman, R.R., Fefferman, C.: Weighted norm inequalities for maximal functions and singular integrals. Stud. Math. 51(3), 241-250 (1974)

4. Garcia-Cuerva, J., Rubio de Francia, J.L.: Weighted Norm Inequalities and Related Topics. North-Holland Mathematics Studies, vol. 116 (1985)

5. Gürbüz, F:: On the behaviors of rough multilinear fractional integral and multi-sublinear fractional maximal operators both on product $L^{p}$ and weighted $L^{p}$ spaces (2018). arXiv:1603.03466v2

6. Hu, Y., Wang, Y.S.: Oscillation and variation inequalities for the multilinear singular integrals related to Lipschitz functions. J. Inequal. Appl. 2017, Article ID 292 (2017)

7. Komori, Y., Shirai, S.: Weighted Morrey spaces and a singular integral operator. Math. Nachr. 282(2), $219-231$ (2009)

8. Morrey, C.B.: On the solutions of quasi-linear elliptic partial differential equations. Trans. Am. Math. Soc. 43, 126-166 (1938)

9. Muckenhoupt, B.: Weighted norm inequalities for the Hardy maximal function. Trans. Am. Math. Soc. 165, 207-226 (1972)

10. Muckenhoupt, B., Wheeden, R.L.: Weighted norm inequalities for singular and fractional integrals. Trans. Am. Math. Soc. 161, 249-258 (1971)

11. Muckenhoupt, B., Wheeden, R.L.: Weighted norm inequalities for fractional integrals. Trans. Am. Math. Soc. 192, 261-274 (1974)

12. Zhang, J., Wu, H.X.: Oscillation and variation inequalities for singular integrals and commutators on weighted Morrey spaces. Front. Math. China 11(2), 423-447 (2016) 